\title{
Prosthetic valve endocarditis resulting from Nocardia asteroides
}

\author{
R. H. FALK1, FRANCES R. DIMOCK ${ }^{2}$, AND J. SHARKEY \\ From the Departments of Cardiology and Microbiology, Dudley Road Hospital, Birmingham, \\ and Burton General Hospital, Burton-on-Trent
}

SUMMARY Nocardia asteroides was isolated from the prosthetic valve of a 64-year-old woman who had died of endocarditis after aortic valve replacement. Multiple blood cultures had all been negative.

This organism is increasingly being recognised as a pathogen and is usually sensitive to sulphonamides. These should be tried in culture negative endocarditis unresponsive to standard therapy.

\section{Case report}

A 64-year-old woman had an aortic valve replacement with a Björk-Shiley prosthesis for severe calcific aortic valve disease. Immediate postoperative recovery was good and the prosthetic valve functioned well.

Six months after operation she experienced anorexia, weight loss, and night sweats. She was admitted to The General Hospital, Burton-onTrent, 3 weeks after the onset of symptoms.

Examination revealed 3 splinter haemorrhages, no clubbing, clear prosthetic heart sounds, no hepatosplenomegaly, and normal urine. There was an intermittent pyrexia ranging from $36^{\circ} \mathrm{C}$ to $38.5^{\circ} \mathrm{C}$. Haemoglobin $13.0 \mathrm{~g} / \mathrm{dl}$. Leucocytes 15600 ( $80 \%$ neutrophils). Erythrocyte sedimentation rate $101 \mathrm{~mm} / \mathrm{h}$. Urea and electrolytes and liver function tests normal. Chest $x$-ray film showed cardiomegaly and pulmonary congestion but no evidence of focal infection.

Blood cultures were sterile, but in view of the possibility of an infected prosthesis she was started on gentamicin and ampicillin. Despite this therapy, her pyrexia persisted, the erythrocyte sedimentation rate remained high, and she became progressively more anaemic.

Three weeks after admission, she developed aortic systolic and diastolic murmurs and was transferred to Dudley Road Hospital, Birmingham, under the care of Dr J. Mackinnon.

On examination she was anaemic with splinter haemorrhages, collapsing pulse, cardiomegaly, and

\footnotetext{
'Present address: Cardiology Department, King's College Hospital, London SE5.

'Present address: Department of Microbiology, Birmingham General Hospital.
}

murmurs of aortic regurgitation. Blood pressure was $120 / 45 \mathrm{mmHg}$. She had a moderate left hemiplegia and bilateral papilloedema.

Haemoglobin $7.9 \mathrm{~g} / \mathrm{dl}$. Leucocytes 9000 . Blood cultures negative. She rapidly deteriorated, became comatose, and died.

NECROPSY (S. M. Abraham)

This showed left ventricular hypertrophy and fibrinous covering of the prosthetic valve on which small vegetations were present. A suture had become detached, giving rise to paraprosthetic leak (Fig. 1).

There was splenomegaly and extensive haemorrhage into the right cerebral hemisphere (Fig. 2). A cystic lesion was present in the left kidney.

Culture of the vegetations yielded colonies of Gram-positive branching filaments identified as Nocardia asteroides. The organism was resistant to both gentamicin and ampicillin but sensitive to sulphonamides.

Histology of the vegetations and of the kidney showed the same organism but it was not seen on histology of the brain or spleen.

A strongly positive precipitin reaction of the patient's serum, taken before death, was obtained with micropolysporon antigen (farmer's lung reaction) due to cross-reacting antibodies and the precipitin test for Nocardia asteroides was also strongly positive.

\section{Comment}

Infection of a prosthetic heart valve is a wellrecognised complication occurring either shortly after operation or several months later (Dismukes et al., 1973). The spectrum of infecting organisms differs slightly from the usual in infective endo- 


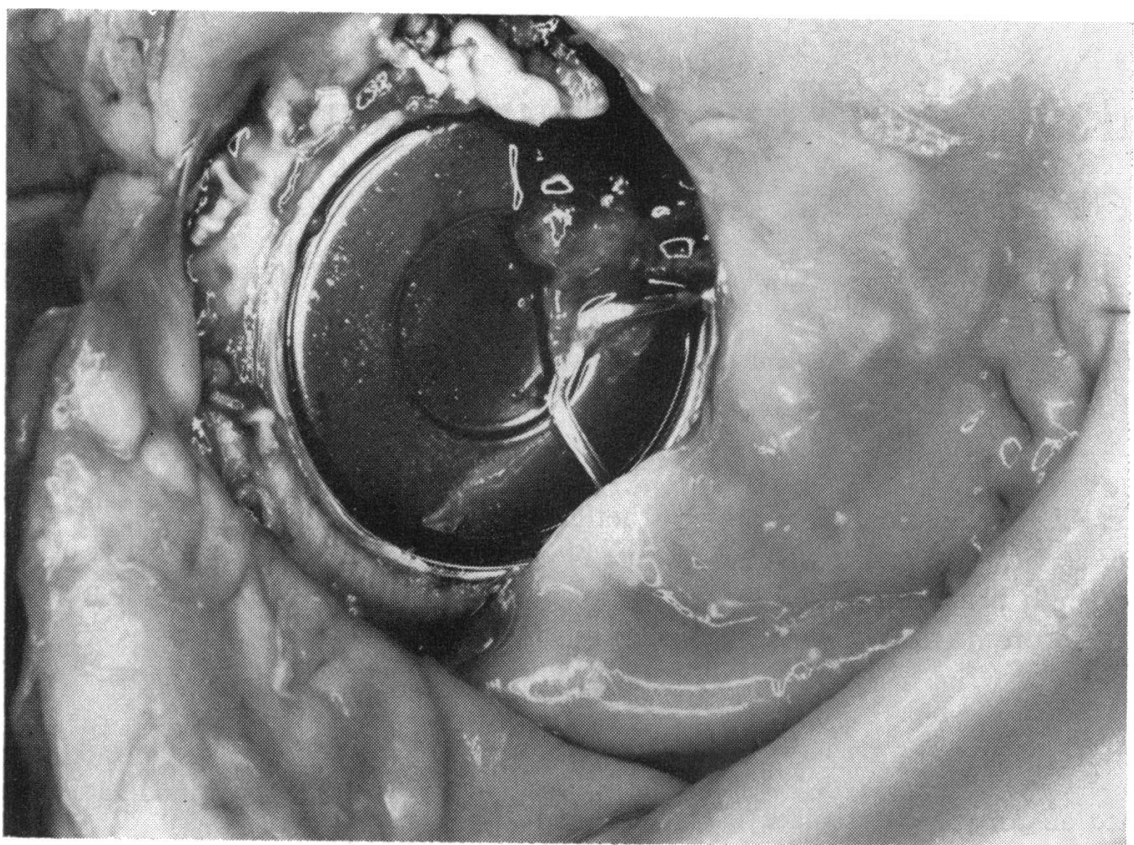

Fig. 1 Björk-Shiley prosthesis showing vegetations.

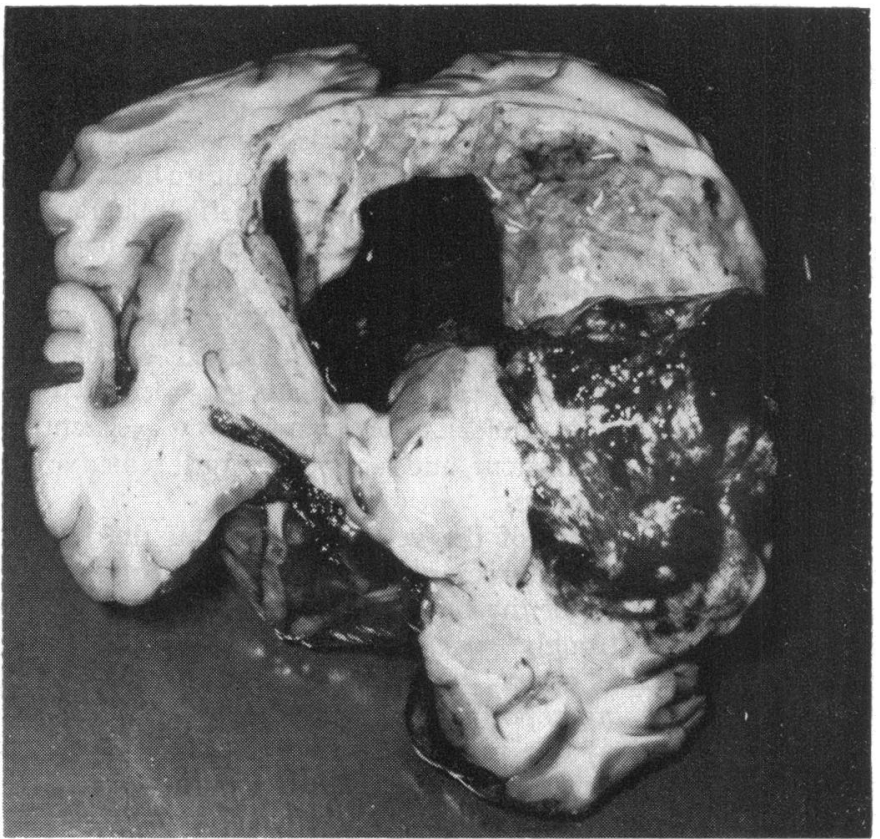

Fig. 2 Brain-showing extensive cerebra haemorrhage extending into the ventricles.

carditis with staphylococci predominating but with a proportion of Gram-negative or fungal organisms (Slaughter et al., 1973).

Nocardia asteroides, a Gram-positive fungus, is now increasingly recognised as a pathogen in immunosuppressed patients and those with lymphoreticular disorders, and pulmonary involvement $\varrho$ occurs in over 70 per cent of patients (Palmer et al., 1974). It has only been reported once previously 8 as a cause of infection on a prosthetic valve 
(Vlachakis et al., 1973). In the present case, and in the one previously reported, there was no clinical or radiological pulmonary involvement. Blood cultures were negative in both cases but the organism has been isolated from skin lesions (which were not present in our patient).

Despite the fact that widespread dissemination of the organism, particularly to the central nervous system, is common, treatment of systemicnocardiasis with sulphonamides has been very successful even in patients with severe immunological impairment (Palmer et al., 1974).

Although definitive diagnosis depends upon isolation of the organism, this may be difficult as blood cultures are frequently negative. Precipitin tests may be helpful and a trial of sulphonamides in a patient with the clinical features of endocarditis, with negative blood cultures, unresponsive to standard therapy, may be life-saving.
We thank Dr J. Mackinnon for permission to publish the case and Dr S. M. Abraham for the necropsy and histology reports.

\section{References}

Dismukes, W. E., Karchmer, A. W., Buckley, M. J., Austen, W. G., and Swartz, M. N. (1973). Prosthetic valve endocarditis-analysis of 38 cases. Circulation, 48, 365-377.

Palmer, D. L., Harvey, R. L., and Wheeler, J. K. (1974). Diagnostic and therapeutic considerations in nocardia asteroides infection. Medicine, 53, 391-401.

Slaughter, L., Morris, J. E., and Starr, A. (1973). Prosthetic valvular endocarditis. A 12-year review. Circulation, 47, 1319-1326.

Vlachakis, N. D., Gazes, P. C., and Hairston, P. (1973). Nocardial endocarditis following mitral valve replacement. Chest, 63, 276-278.

Requests for reprints to Dr R. H. Falk, Cardiac Department, King's College Hospital, London SE5. 